\title{
Reversible adiabatic temperature changes at the magnetocaloric and barocaloric effects in $\mathrm{Fe}_{49} \mathrm{Rh}_{51}$
}

\author{
Enric Stern-Taulats, ${ }^{1}$ Adrià Gràcia-Condal, ${ }^{1}$ Antoni Planes, ${ }^{1}$ Pol Lloveras, ${ }^{2}$ Maria Barrio, ${ }^{2}$ \\ Josep-Lluís Tamarit, ${ }^{2}$ Sabyasachi Pramanick, ${ }^{3}$ Subham Majumdar, ${ }^{3}$ and Lluís Mañosa ${ }^{1}$ \\ ${ }^{1}$ Departament d'Estructura i Constituents de la Matèria, Facultat de Física, Universitat de Barcelona, \\ Martí i Franquès, 1, E-08028 Barcelona, Catalonia, Spain \\ ${ }^{2}$ Grup de Caracterització de Materials, Departament de Física i Enginyeria Nuclear, ETSEIB, \\ Universitat Politècnica de Catalunya, Diagonal 647, 08028 Barcelona, Catalonia, Spain \\ ${ }^{3}$ Department of Solid State Physics, Indian Association for the Cultivation of Science, Jadavpur, \\ Kolkata 700 032, India
}

(Received 22 July 2015; accepted 7 October 2015; published online 15 October 2015)

\begin{abstract}
We report on the adiabatic temperature changes $(\Delta T)$ associated with the magnetocaloric and barocaloric effects in a $\mathrm{Fe}_{49} \mathrm{Rh}_{51}$ alloy. For the magnetocaloric effect, data derived from entropy curves are compared to direct thermometry measurements. The agreement between the two sets of data provides support to the estimation of $\Delta T$ for the barocaloric effect, which are indirectly determined from entropy curves. Large $\Delta T$ values are obtained at relatively low values of magnetic field $(2 \mathrm{~T})$ and hydrostatic pressure $(2.5 \mathrm{kbar})$. It is also shown that both magnetocaloric and barocaloric effects exhibit good reproducibility upon magnetic field and hydrostatic pressure cycling, over a considerable temperature range. (C) 2015 AIP Publishing LLC.

[http://dx.doi.org/10.1063/1.4933409]
\end{abstract}

Fe-Rh alloys with compositions close to the 1:1 stoichiometry transform on cooling from a ferromagnetic (FM) to an antiferromagnetic (AFM) phase close to room temperature. ${ }^{1}$ The transition is first order, it does not involve a change in the crystal symmetry $(\mathrm{CsCl}$ structure, $\mathrm{Pm} 3 \mathrm{~m})$, but the unit cell isotropically shrinks, giving rise to a relative volume change of about $1 \%$. The sensitivity of the transition to magnetic field and mechanical stress along with the latent heat of the phase transition gives rise to a series of caloric effects in this compound. Interestingly, although Fe-Rh was the first material in which giant magnetocaloric ${ }^{2}$ and elastocaloric $^{3}$ effects were reported, the interest in this alloy remained very low because it was believed that these effects were not reproducible. 4

In the recent years, however, there has been a renewed interest in the study of Fe-Rh. ${ }^{5-11}$ On the one hand, it has been evidenced that in accurately prepared samples, the entropy change associated with the magnetocaloric effect exhibits good reproducibility upon magnetic field cycling. ${ }^{10,12}$ It has also been shown ${ }^{10}$ recently that in addition to the aforementioned magnetocaloric and elastocaloric effects, the alloy also exhibits a giant barocaloric effect, associated with the application of hydrostatic pressure. The strong sensitivity of the transition temperature to the external fields (magnetic and mechanical) confers to this alloy outstanding magnetocaloric and barocaloric strengths in comparison to other materials, ${ }^{13}$ which make it particularly interesting for cooling applications at low fields. It is also worth mentioning that understanding the strong coupling between several degrees of freedom (magnetic, structural, and electronic) represents a challenge from a fundamental point of view. $5,7,9,11$

A complete assessment of the caloric performances of a given caloric material requires knowledge of both the isothermal entropy change and the adiabatic temperature change. ${ }^{13-15}$ In addition, the reversibility of the effect under a cyclic variation of the external field must also be considered. ${ }^{16-20}$ In a previous work, ${ }^{10}$ we reported on the isothermal entropy change of $\mathrm{Fe}_{49} \mathrm{Rh}_{51}$. We provided the first evidence of the existence of giant barocaloric effects and we proved that the values for the entropy change in both magnetocaloric and barocaloric effects were reproducible upon field cycling. In the present paper, we address the adiabatic temperature change in both caloric effects by means of direct and indirect measurement techniques. The values for the adiabatic temperature change found for low fields are significantly large, giving rise to outstanding caloric strengths also in relation to the adiabatic temperature changes. Furthermore, an excellent reproducibility upon magnetic field and hydrostatic pressure cycling has been found.

A polycrystalline sample of nominal composition $\mathrm{Fe}_{49} \mathrm{Rh}_{51}$ was prepared by arc melting the pure metals under argon atmosphere in a water-cooled $\mathrm{Cu}$ crucible. For homogeneity, the sample was remelted several times turning the ingot back to back. Next, the ingot was vacuum sealed in a quartz tube and annealed at $1100{ }^{\circ} \mathrm{C}$ for $72 \mathrm{~h}$ followed by a furnace cooling to room temperature. From the ingot, a sample with the shape of a truncated half ellipsoid $\left(\sim 50 \mathrm{~mm}^{3}\right)$ was cut using a low speed diamond saw. Present sample has the same nominal composition than the sample studied in Ref. [10], and the transition temperatures are coincident within experimental error. However, conventional DSC measurements render a value for the transition entropy change $\left(\Delta S_{t}=11.1 \mathrm{~J} / \mathrm{kg} \mathrm{K}\right)$ that is slightly lower than that of the previously studied sample $\left(\Delta S_{t}=12.5 \mathrm{~J} / \mathrm{kg} \mathrm{K}\right)$.

For direct measurements of the temperature change, a fine gauge $\mathrm{K}$ thermocouple $(0.075 \mathrm{~mm}$ diameter) was embedded into a hole drilled to the sample. A good thermal contact between the sample and the thermocouple was improved by means of a conductive paste. The sample was put in contact 


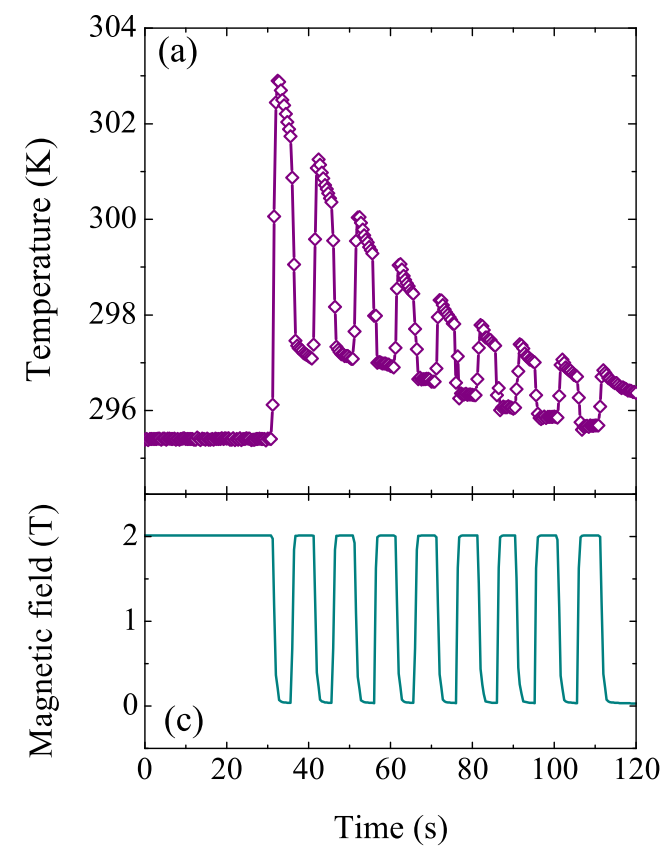

with one of the faces of a Peltier module to control the operating temperature. The opposite face of the Peltier module was in contact with the upper end of a copper bar, which acted as a heat sink. A micro-Hall sensor was attached next to the sample to monitor the magnetic field. The ensemble was wrapped together with Teflon tape and it was placed into the gap $(1.2 \mathrm{~cm})$ of an electromagnet where fields up to $2 \mathrm{~T}$ can be applied at rates of $1.5 \mathrm{~T} / \mathrm{s}$. The bottom end of the bar was immersed into an iced water bath. The operating temperature was fixed by tuning the current through the Peltier element, and the output from the thermocouple and Hall sensor was read at a rate of $2.5 \mathrm{~Hz}$ by two multimeters, which were connected to a computer. Adiabaticity relies on the ratio between the characteristic time constant associated with the application (or removal) of the field $\left(\tau_{1}\right)$ and the time constant associated with heat exchange between sample and surroundings $\left(\tau_{2}\right)$. In our case, $\tau_{1}<10^{-2} \tau_{2}$, which suggests a quite good adiabaticity in our measurements.

Taking into account that in Fe-Rh magnetic field stabilizes the high temperature FM phase (as heating does) which results in an inverse magnetocaloric effect, the following protocols were followed to carry out the adiabatic temperature measurements. Heating protocol: The sample was first cooled in the absence of magnetic field down to a temperature where it was fully transformed into the AFM phase. After allowing for thermal equilibrium, the sample was heated up to the desired measurement temperature. After reaching the new thermal equilibrium at the operating temperature, a sequence of 10 magnetic field cycles between 0 and $2 \mathrm{~T}$ was conducted. Cooling protocol: The sample was heated under an applied field of $2 \mathrm{~T}$ up to a temperature where it was fully in the FM phase. After thermal equilibrium, it was cooled (under magnetic field) down to the desired operating temperature. Once the sample had reached the new thermal equilibrium, the magnetic field was cycled (10 times) from $2 \mathrm{~T}$ to 0 . Figure 1 illustrates examples of the temperature and magnetic field measurements recorded during cooling (Figs. 1(a) and $1(\mathrm{c})$ ) and heating (Figs. 1(b) and 1(d)) protocols. No noticeable traces of cracking were observed on the specimen after the sample was cycled through the AFM/FM transition many times (between 50 and 100 cycles).

Figure 2(a) compiles the whole set of results measured at different operating temperatures. Solid symbols correspond to

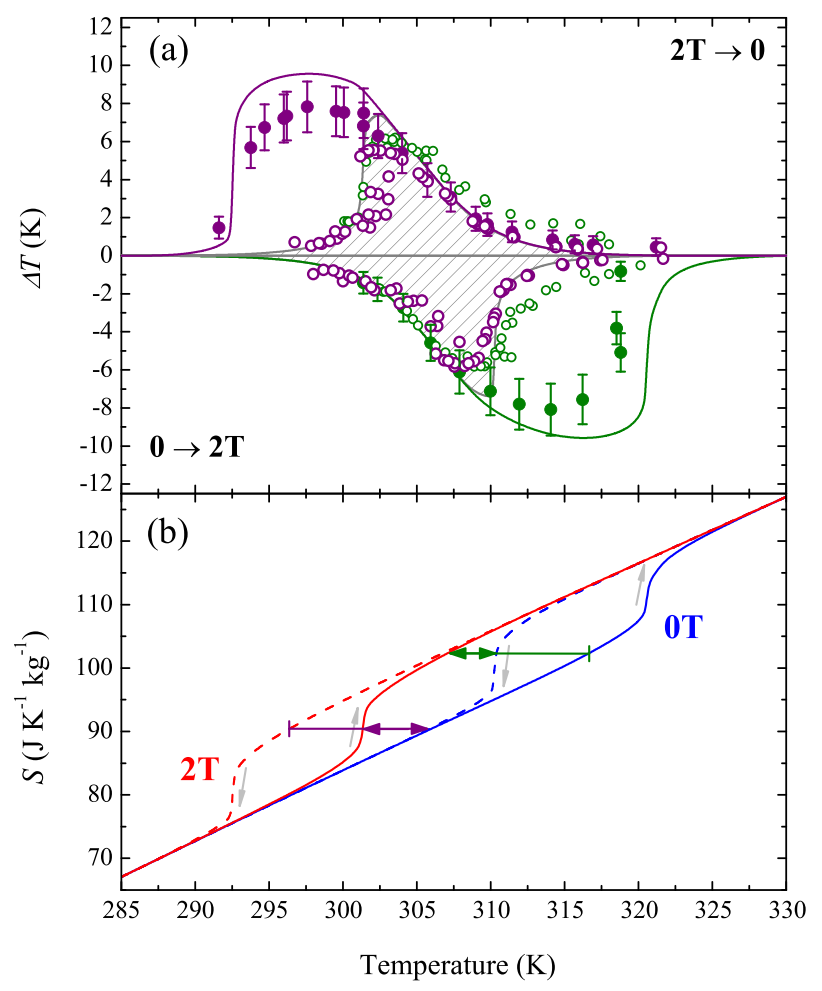

FIG. 2. (a) Adiabatic temperature change corresponding to the application and removal of a $2 \mathrm{~T}$ magnetic field. Solid symbols correspond to the first application (green color) and removal (violet color) of the field. Open symbols correspond to the subsequent field cycling for cooling (violet symbols) and heating (green symbols) protocols. Lines correspond to the values computed indirectly from entropy curves. The shaded area indicates the reversibility region. (b) Entropy as a function of temperature at zero (blue color) and $2 \mathrm{~T}$ (red color) magnetic fields. Dashed lines correspond to cooling and solid lines to heating runs. Horizontal arrows indicate the adiabatic temperature changes computed from these curves. 
the first application (green circles) and removal (violet circles) of the field, while open symbols stand for the data recorded upon successive field cycling. For the sake of clarity, error bars are only displayed for the data corresponding to the first application and removal of the field, and are estimated from reproducibility of three independent measurements. In accordance with the inverse nature of the magnetocaloric effect in $\mathrm{Fe}-\mathrm{Rh}$, application of magnetic field results in cooling while removal of the field heats the sample. The maximum values found for the first application (and first removal) of the field are very large $(|\Delta T|=8 \mathrm{~K})$, which result in a magnetocaloric strength which is among the largest values reported for giant magnetocaloric materials. ${ }^{13}$ Upon successive cycling of the magnetic field, $|\Delta T|$ decreases but still large values $(|\Delta T|=6 \mathrm{~K})$ are obtained. It is also worth noting that the reversibility region (indicated by the shaded area) spans over a considerable temperature interval of around $20 \mathrm{~K}$.

Indirect determination of the adiabatic temperature change can be computed by subtraction of the entropy curves $S(T, Y)$ at different values of the external field $Y$ (magnetic, $Y=H$ or pressure, $Y=p$ ) which are obtained from calorimetric measurements under external field. At temperatures above and below the first order phase transition, $S(T, Y)$ are obtained from specific heat $C$ data, while within the transition region (between $T_{1}$ and $T_{2}$ ), accurate measurements come from differential scanning calorimetry (DSC) under field. The entropy curves (referenced to the value at a given temperature $T_{0}$ ) for heating runs are then computed as

$$
S(T, Y)= \begin{cases}\int_{T_{0}}^{T} \frac{C^{A F M}}{T} d T & T \leq T_{1} \\ S\left(T_{1}, Y\right)+\int_{T_{1}}^{T} \frac{1}{T}\left(C+\frac{d Q}{d T}\right) d T & T_{1}<T \leq T_{2} \\ S\left(T_{2}, Y\right)+\int_{T_{2}}^{T} \frac{C^{F M}}{T} d T & T_{2}<T,\end{cases}
$$

where $\frac{d Q}{d T}=\frac{\dot{Q}}{\dot{T}}$ with $\dot{Q}$ being the heat flux measured by DSC and $\dot{T}$, the heating rate. $C^{A F M}$ and $C^{F M}$ are, respectively, the specific heat of the AFM and FM phases and $C=x C^{A F M}$ $+(1-x) C^{F M}$ with $x$ the fraction in the AFM phase. For $\mathrm{Fe}-\mathrm{Rh}$, within the transition region, $C \approx C^{A F M} \approx C^{F M}$. By appropriate change of the integration limits, an equivalent expression is used for cooling runs.

By using our previous DSC data under magnetic field and under hydrostatic pressure, ${ }^{10}$ and reported values of $C$ in the AFM and FM phases, ${ }^{7}$ we have computed the entropy curves $S(T, H)$ at atmospheric pressure and fields for $\mu_{0} H=0$ and $2 \mathrm{~T}$, and $S(T, p)$ in the absence of magnetic field for atmospheric pressure (taken as $p=0$ ) and $p=2.5 \mathrm{kbar}$. In our calculations we have made the usual assumption that beyond the transition region $C$ does not significantly depend upon magnetic field and pressure. Furthermore, in order to account for the slight difference in the transition entropy change between present sample and that studied in, ${ }^{10}$ DSC data have been scaled by a factor 0.9 . Results are shown in Figures 2(b) and 3(b) where dashed lines correspond to

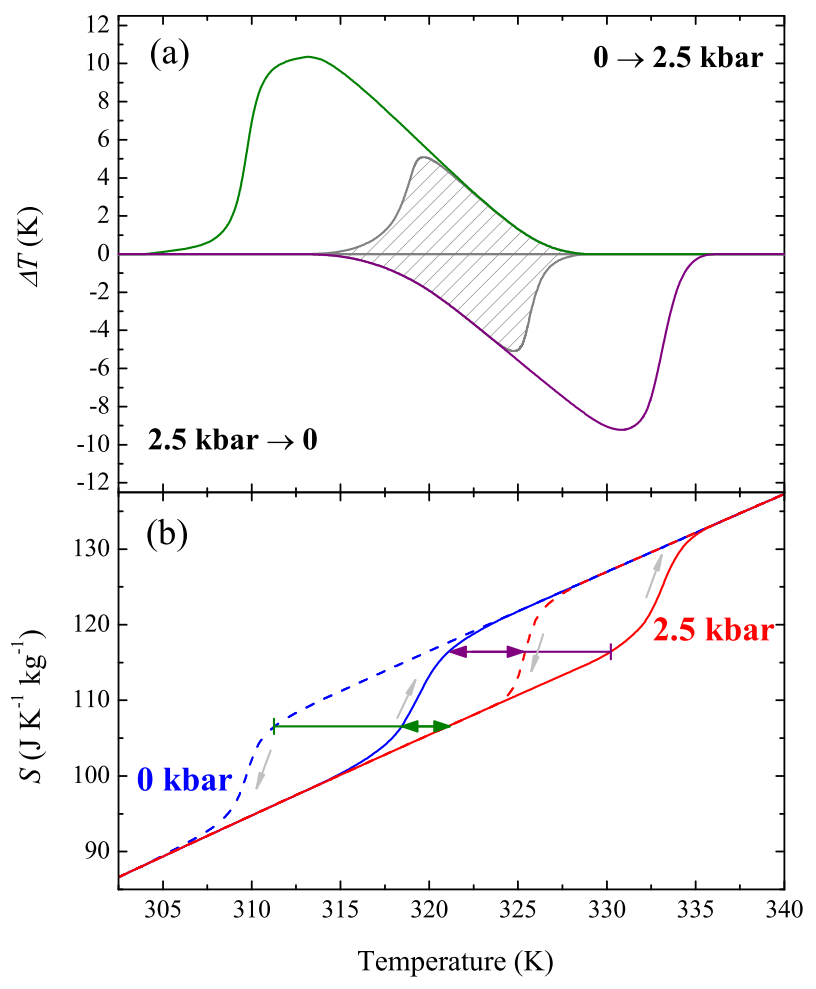

FIG. 3. (a) Adiabatic temperature change corresponding to the application and removal of a $2.5 \mathrm{kbar}$ hydrostatic pressure computed indirectly from entropy curves. The shaded area indicates the reversibility region. (b) Entropy as a function of temperature at atmospheric pressure $(p=0$, blue color) and at $p=2.5 \mathrm{kbar}$ (red color). Dashed lines correspond to cooling runs and solid lines to heating runs. Horizontal arrows indicate the adiabatic temperature changes computed from these curves.

cooling curves and solid lines, to heating curves. Subtraction of the curves provides the estimated adiabatic temperature changes, as indicated by the horizontal arrows, and left/right arrows indicate the reversible values corresponding to successive field cycling. Results are shown in Figures 2(a) and 3 (a) as solid lines where the reversibility region corresponds to the shaded region.

For the magnetocaloric effect, there is a good coincidence between the two sets of data although the values for the direct measurements of $\Delta T$ are systematically lower (around 10\%) than those computed from the entropy curves. Such a small difference can be attributed to a lack of perfect adiabaticity in our experimental set-up, and to a non-ideal thermal contact between the sample and the thermocouple. In the case of the barocaloric effect, it is shown that application of pressure heats up the sample while the sample cools down when pressure is released, in concordance with the conventional nature of the barocaloric effect in Fe-Rh. The maximum values $(|\Delta T| \sim 10 \mathrm{~K})$ are very large for a relatively small pressure of $2.5 \mathrm{kbar}$. These values are significantly larger than those reported (or estimated) for other barocaloric materials. ${ }^{13,21-24}$ It is also worth noting that upon pressure cycling $|\Delta T|$ remain at relative large values of $\sim 4 \mathrm{~K}$ over a temperature span of $\sim 10 \mathrm{~K}$.

Recently, the magnetocaloric and barocaloric effects in caloric materials have been studied by means of a mean-field model which includes magnetovolumic effects. ${ }^{11}$ The model has been applied to the specific case of Fe-Rh and the 
predicted adiabatic temperature changes are in qualitative agreement with present experimental data. The predicted values, however, are lower than the experimental ones, and the discrepancy is attributed to the fact that the model does not properly take into account the electronic contribution to the total entropy change. No quantitative prediction is made for the adiabatic temperature change at the barocaloric effect, but the model reproduces the conventional nature of the effect, and it is expected that the general trends derived from that model will agree with present experiments.

In summary, we have determined the adiabatic temperature changes associated with the magnetocaloric and barocaloric effects in Fe-Rh. Large $|\Delta T| \sim 8-10 \mathrm{~K}$ values have been found for relatively low values of magnetic field ( $2 \mathrm{~T}$ ) and hydrostatic pressure $(2.5 \mathrm{kbar})$. It has been shown that both magnetocaloric and barocaloric effects are reproducible upon magnetic field and pressure cycling, over considerable temperature spans $\left(T_{\text {span }} \sim 10-15 \mathrm{~K}\right)$. The maximum value for the adiabatic temperature change obtained under cycling reduces to $|\Delta T| \sim 4-6 \mathrm{~K}$, which is still a significantly large value. We recently reported that $\mathrm{Fe}-\mathrm{Rh}$ exhibits outstanding values for the magnetocaloric and barocaloric strengths in terms of the isothermal entropy change. Present data show that this alloy also features large caloric strengths in relation to adiabatic temperature changes. Although the high cost of $\mathrm{Fe}-\mathrm{Rh}$ makes it difficult to envisage large scale applications, the alloy appears as a promising candidate for small scale solid-state cooling at low external stimuli.

We acknowledge financial support from CICyT (Spain), Project Nos. MAT2013-40590-P and FIS2014-54734-P, and from the Joint-Indo-Spanish project, DIGICyT (Spain) Project No. PRI-PIBIN-20-11-0780 and DST (India) Project No. DST/INT/P-39/11. E.S.-T. acknowledges support from AGAUR (Catalonia), and P.L., support from SUR (DEC, Catalonia).
${ }^{1}$ M. Fallot, Ann. Phys. (Paris) 10, 291 (1938).

${ }^{2}$ S. Nikitin, G. Myalikgulyev, A. M. Tishin, M. P. Annaorazov, K. A. Asatryan, and A. L. Tyurin, Phys. Lett. 148, 363 (1990).

${ }^{3}$ S. Nikitin, G. Myalikgulyev, M. P. Annaorazov, A. L. Tyurin, R. W. Myndev, and S. A. Akopyan, Phys. Lett. 171, 234 (1992).

${ }^{4}$ V. Franco, J. S. Blázquez, B. Ingale, and A. Conde, Annu. Rev. Mater. Res. 42, 305 (2012).

${ }^{5}$ S. O. Mariager, F. Pressacco, G. Ingold, A. Caviezel, E. Möhr-Vorobeva, P. Beaud, S. L. Johnson, C. J. Milne, E. Mancini, S. Moyerman et al., Phys. Rev. Lett. 108, 087201 (2012).

${ }^{6}$ A. X. Gray, D. W. Cooke, P. Krüger, C. Bordel, A. M. Kaiser, S. Moyerman, E. E. Fullerton, S. Ueda, Y. Yamashita, A. Gloskovskii et al., Phys. Rev. Lett. 108, 257208 (2012).

${ }^{7}$ D. W. Cooke, F. Hellman, C. Baldasseroni, C. Bordel, S. Moyerman, and E. E. Fullerton, Phys. Rev. Lett. 109, 255901 (2012).

${ }^{8}$ P. M. Derlet, Phys. Rev. B 85, 174431 (2012).

${ }^{9}$ J. B. Staunton, R. Banerjee, M. dos Santos Dias, A. Deak, and L. Szunyogh, Phys. Rev. B 89, 054427 (2014).

${ }^{10}$ E. Stern-Taulats, A. Planes, P. Lloveras, M. Barrio, J. L. Tamarit, S. Pramanick, S. Majumdar, C. Frontera, and L. Mañosa, Phys. Rev. B 89, 214105 (2014).

${ }^{11}$ E. Mendive-Tapia and T. Castán, Phys. Rev. B 91, 224421 (2015).

${ }^{12}$ M. Manekar and S. B. Roy, J. Phys. D: Appl. Phys. 41, 192004 (2008).

${ }^{13}$ X. Moya, S. Kar-Narayan, and N. D. Mathur, Nat. Mater. 13, 439 (2014).

${ }^{14}$ K. Sandeman, Scr. Mater. 67, 566 (2012).

${ }^{15}$ L. Mañosa, A. Planes, and M. Acet, J. Mater. Chem. A 1, 4925 (2013).

${ }^{16}$ I. Titov, M. Acet, M. Farle, D. González-Alonso, L. Mañosa, A. Planes, and T. Krenke, J. Appl. Phys. 112, 073914 (2012).

${ }^{17}$ K. P. Skokov, K. H. Muller, J. D. Moore, J. Liu, A. Y. Karpenkov, M. Krautz, and O. Gutfleish, J. Alloys Compd. 552, 310 (2013).

${ }^{18}$ B. Emre, S. Yüce, E. Stern-Taulats, A. Planes, S. Fabbrici, F. Albertini, and L. Manosa, J. Appl. Phys. 113, 213905 (2013).

${ }^{19}$ E. Stern-Taulats, P. O. Castillo-Villa, L. Mañosa, C. Frontera, S. Pramanick, S. Majumdar, and A. Planes, J. Appl. Phys. 115, 173907 (2014).

${ }^{20}$ F. Gillou, G. Porcari, H. Yibole, N. van Dijk, and E. Brück, Adv. Mater. 26, 2671 (2014).

${ }^{21}$ L. Mañosa, D. González-Alonso, A. Planes, E. Bonnot, M. Barrio, J. L. Tamarit, S. Aksoy, and M. Acet, Nat. Mater. 9, 478 (2010).

${ }^{22}$ L. Mañosa, D. González-Alonso, A. Planes, M. Barrio, J. L. Tamarit, I. S. Titov, M. Acet, A. Bhattacharyya, and S. Majumdar, Nat. Commun. 2, 595 (2011).

${ }^{23}$ S. Yüce, M. Barrio, B. Emre, E. Stern-Taulats, A. Planes, J. L. Tamarit, Y. Mudryk, K. A. Gschneidner, V. K. Pecharsky, and L. Mañosa, Appl. Phys. Lett. 101, 071906 (2012).

${ }^{24}$ D. Matsunami, A. Fujita, K. Takenaka, and M. Kano, Nat. Mater. 14, 73 (2014). 